\title{
Gurene Utilitarian Basketry Arts: A Critical Discourse
}

\author{
Rolland Wemegah $^{1 *} \quad$ Dr. Steve Kquofi ${ }^{2} \quad$ Dr. Eric Appau Asante ${ }^{2}$ \\ 1.Industrial Art Department, Bolgatanga Technical University, P. O. Box 767, Bolgatanga- U/E Region, Ghana \\ 2.Department of Educational Innovations in Science and Technology, Kwame Nkrumah University of Science \\ and Technology, P. O. Box UP 79, Kumasi - Ashanti Region, Ghana
}

\begin{abstract}
The Gurene people in the Upper East Region of Ghana have a variety of notable utilitarian basketry articles which are used in many cultural applications in the Gurene society. These category of basketry articles, even though very important in the day-to-day activities of the Gurene people, are not extensively researched into. This study sought to identify, describe and underpin the functions of these basketry articles, in order to underpin their socio-cultural relevance among the Gurene people. Ethnographic research design was used. 85 respondents were sampled for the study using snowball and purposive sampling techniques. Primary data was collected using observations, interviews and focus group discussions and analysed thematically, using NVivo 9 qualitative data analysis package. The findings showed that the basketry articles are employed in agricultural and culinary activities such as harvesting, carting of farm produce, storage of grains, rearing of livestock, winnowing, and food preparations. Also, some are utilised in burial and funeral rites, expended as architectural accoutrements, and applied as containers and carriers which are used in selling diverse items in the Gurene society.
\end{abstract}

Keywords: Gurene basketry, baskets, ethnic artefacts, utilitarian basketry arts, fibre arts.

DOI: $10.7176 / \mathrm{ADS} / 80-03$

Publication date: January $31^{\text {st }} 2020$

\section{Introduction}

Basketry arts play varied important roles in the day-to-day activities of many civilizations around the globe. Indeed, basketry artefacts are considered essential utilities, which are needed for the proper functioning of many agrarian societies around the world. Among the Gurene people of the Upper East Region of Ghana, a cornucopia of basketry wares could be detected in virtually every nook and cranny of the society. These intricately crafted articles are used in many cultural applications by the Gurene people.

The term "basketry" is used by experts as an umbrella term to encompass varied woven articles crafted from assorted vegetative fibres of limited length (Wendrich, 1991). Though basketry products may be quite mundane, (Bunn 2014) explains that they are important articles that aid in throwing light on the socio-cultural dynamics of the community that produces them. Guss (1989) buttresses the above by succinctly revealing that basketry objects are "portraits of the society that inspired them".

Basketry generally, is believed to be one of the essential ancient arts which can be associated with almost all aboriginal groupings. They have been utilized as containers, architectural accessories, fashion accents, traps, cages, boats, hats, beehives, furniture, amour, chariots, and boats in many cultures (Kaller 2012; Kepe, 2003).

Historical evidence of basketry items is limited worldwide due to their perishable nature. Despite the above limitation, experts believe basketry is the earliest craft produced by man (Bizzari, 2012; Pollock, 1993). Archaeological evidence from Egypt, Peru, Southern Spain, Switzerland and Jarmo, indicates that the art of basketry was known to civilizations of the Neolithic period (Singh, 2016; Balfet, 2014; Bizzari, 2012). Appreciable basketry fragments discovered in the Middle East by archaeologists points to the fact that the art form was practiced by many cultures about 7,000 years ago (Singh \& Davidson 2016). Other basketry remains dated to 7000 B. C. were equally discovered near Dangercave (Wendover), in Utah, indicating their production and use by early civilizations in the area (Harvey, 1975).

Because all primeval cultures required containers to gather, transport and store various agrarian items, it is the contention of some researchers that no single human culture can lay claim to the invention of basketry (Crowford, 2003). Crowford believes basketry articles might have been developed independently by various civilizations concurrently.

\subsection{Research Objective}

The general objective of the research was to study the basketry arts of the Gurene people. Specifically, the research sought to identify, describe, and underpin the functions of core utilitarian basketry articles produced and used in diverse sectors of the indigenous Gurene society.

\subsection{Problem Statement}

Gurene utilitarian basketry objects are ethnographic artefacts produced purposely for use in the Gurene society. Their cultural functions are quite different from the more ubiquitous "Bolga baskets", which are a wide range of 
trade basketry articles manufactured for export, or sold to tourists, and many middle- and high-class collectors within, and outside Ghana. The utilitarian basketry articles could be found in every Gurene community, and are employed in many cultural activities within the Gurene socio-cultural environment. Despite their enormous socio-cultural essentiality, very limited literature could be found on these class of basketry objects, due to the scanty studies carried out on them. The ethnographic work of Smith (1978), which studied some of these basketry products in the Gurene community is quite limited in scope. Many of the utilitarian basketry artefacts were therefore not studied by the author.

It is common knowledge that basketry is presently experiencing significant changes around the world. Many of its traditional functions are being fulfilled by manufactured products such as card boxes, wooden, enamel, plastic and metal containers, as well as paper and plastic carrier bags (Bunn 2014). This development, according to Bunn, has led to the obliteration of basketry traditions in some societies. There is therefore an urgent need to study, document and preserve knowledge relating to these cultural artefacts for posterity.

\section{Methodology}

The data presented below was gathered from a wide geographical area within the Gurene ethnic boundaries, specifically, Bolgatanga, Bongo, and Tallensi communities in the Upper East Region of Ghana.

The study made use of qualitative techniques and tools (Domegan \& Fleming, 2007; Denzin \& Lincoln, 2003) in undertaking the study. Specifically, an ethnographic research design was used. Ethnography is particularly concerned with participating in the activities of a given community. It involves; observing, interpreting and analytically writing about the people and their cultures, rather than testing a hypothesis (Reeves, Kuper \& Hodges, 2008; Marvasti, 2004; Creswell, 2003; Atkinson \& Hammersley, 1994). Using snowball (Lune $\&$ Berg; 2017) and purposive sampling techniques (Cohen, Manion \& Morrison, 2007), 85 respondents were selected for the study. Data were subsequently collected from the respondents using interviews, observations (both participant and non-participant) and focus group discussions. Primary data was collected by the researchers themselves through an interpreter. Various photographs were also taken during the field activities to support the study. The field data was subsequently transcribed, cleaned, and loaded into an NVivo 9 data analysis software programme, and analysed using thematic data analysis technique. Thematic analysis is a rigorous and methodical technique employed in examining and verifying a body of data, to unearth salient themes that can be used in addressing a research problem (Maguire \& Delahunt, 2017).

\section{Findings and Discussions}

The study established the presence of a miscellanea of basketry articles in the Gurene society. These articles are presented under five broad subheadings, namely; containers and carriers, architectural basketry objects, clothing articles, mats, as well as cages and nests.

The research established that basketry objects are produced in virtually every Gurene community, with some of the communities specializing in the production of some specific basketry objects. In the Bolgatanga Municipality, some of the notable basketry production communities are; Bolgatanga, Sumbrungu, Soe, Nyarega, Gambibgo, Zaare, Yikene, Dulugu, Tindomolgo, Yorogo, Zorbisi, and Tanzui. In the Bongo District and its environs, basketry production is prevalent in communities such as; Bongo, Bongo Soe, Zokor, Dua Dooni, Ballugu Vea, Gowrie and Namoo; while communities such as Tongo, Yazore, Winkogo, Sheaga, Gbeogo, Pwalugu, and Kpatia are reputed for being some of the prominent basketry manufacturing centres in the Tallensi District.

\subsection{Containers and Carriers}

Two different classes of container and carriers, specifically; the pio variety of baskets and the piog/ benka baskets are presented under this subheading. The basketry articles are singly described below, and their respective functions duly underpinned.

\subsubsection{Pio Baskets}

Four types of basketry articles were discovered during the study which carry the "pio" nomenclature. These articles were constructed using similar basketry crafting techniques, and are duly presented below in figures 1,2 , 3 and 4 respectively.

The first variety which is shown in figure 1 below, is usually produced with, or without a handle (see figure 1). They have square bases measuring $20 \times 20 \mathrm{~cm}$, with slanted sides towering about $40 \mathrm{~cm}$ (excluding the handle), and circular rims spanning $35 \mathrm{~cm}$ in diameter. The respondents explained that this variety of the pio baskets (contemporary pio baskets) evolved from the older types pictured in figure 2 below. It functions as a utility basket. They are employed in harvesting, transporting, and storing various farm produces. They are also used in storing cooking ingredients as well as utensils such as; pots, bowls, ladles, stirring sticks, and calabashes. 


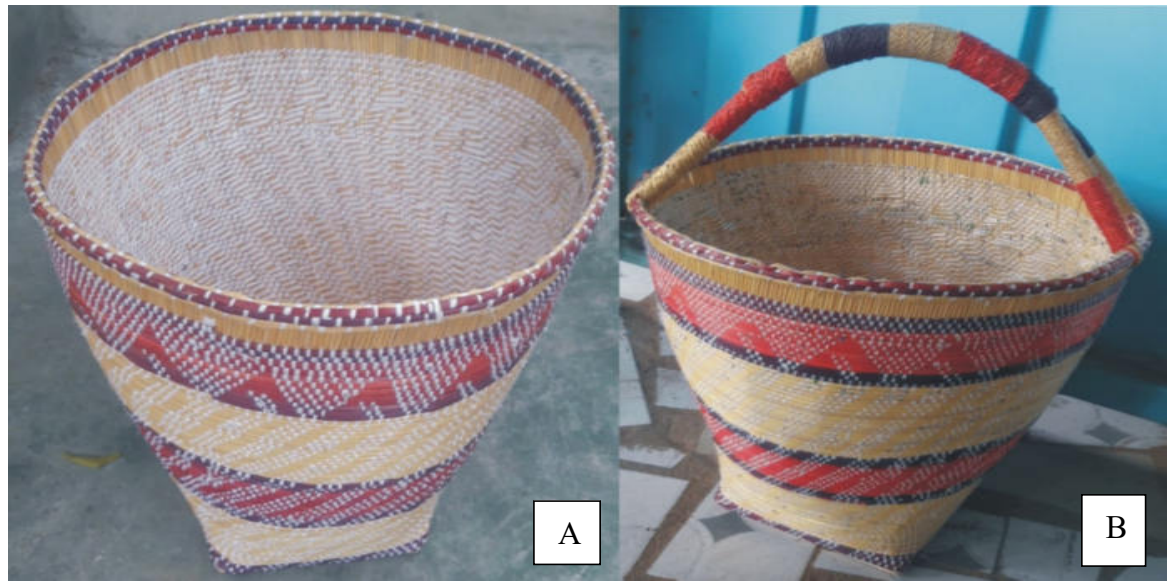

Figure 1. Contemporary pio baskets

The pio baskets captured below in figures 2 " $\mathrm{A}$ " and " $\mathrm{B}$ " are quite different in size. The basket pictured in figure 2 "A" is quite smaller in size. It has a base dimension of $17 \times 17 \mathrm{~cm}$, a height of $27 \mathrm{~cm}$, and a rim dimension of $32 \mathrm{~cm}$ in diameter; while the larger variety shown in figure 2 "B" has a base dimension of $24 \mathrm{x}$ $24 \mathrm{~cm}$, a height of $47 \mathrm{~cm}$, and a rim dimension of $53 \mathrm{~cm}$ in diameter.

Generally, it was explained that the smaller pio basket (figure 2 "A") is mostly used in storing cooking ingredients such as dried fish and meat, salt, spices, and assorted vegetables. Some of the respondents also pointed out that this variety of the pio baskets are also used by women to convey gifts such as bambara beans and shea butter to their in-laws.

The larger variety, (see figure 2 "B") on the other hand, as agreed by the respondents, is normally used in harvesting, transporting, and storing assorted farm produces. They are also applied as winnowing baskets, and sometimes also used in storing cooking utensils.
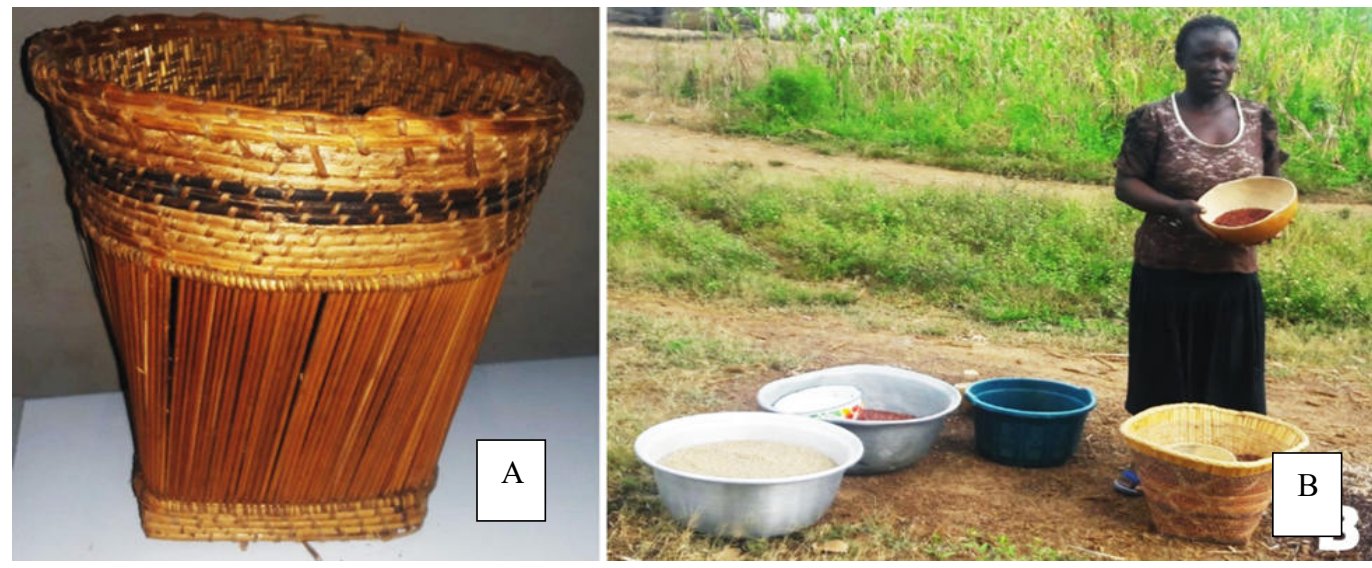

Kure Pio

Figure 2. Smaller pio basket "A", larger pio basket being used in winnowing "B"

Kore pio baskets (see figure 3 below) are the quintessential baskets used in female funeral rites among the Gurene people. Unlike the pio baskets described above, the rims of the kure pio are roughly finished. They equally have kenaf fibres loosely hanging on the body of the baskets, as could be seen in figure 3 below. The baskets usually have square bases that measure about $20 \times 20 \mathrm{~cm}$, slanted sides reaching heights of $29 \mathrm{~cm}$, and rims measuring $47 \mathrm{~cm}$ in diameter.

The kure pio articles are mostly used during the final funeral rites of elderly women. They are normally employed in conveying items such as calabashes, pots, and mats to a footpath leading to the deceased woman's house, during the performance of the funeral rites. The pots and calabashes were subsequently broken, and the basket burnt together with a mat at the above-mentioned location (see figure 3 "B" below). Thereafter, a guinea fowl was sacrificed at the site. The respondents explained that the above rites symbolize the transfer of the pots, calabashes, mat, and basket to the deceased woman for use in the spirit world. 

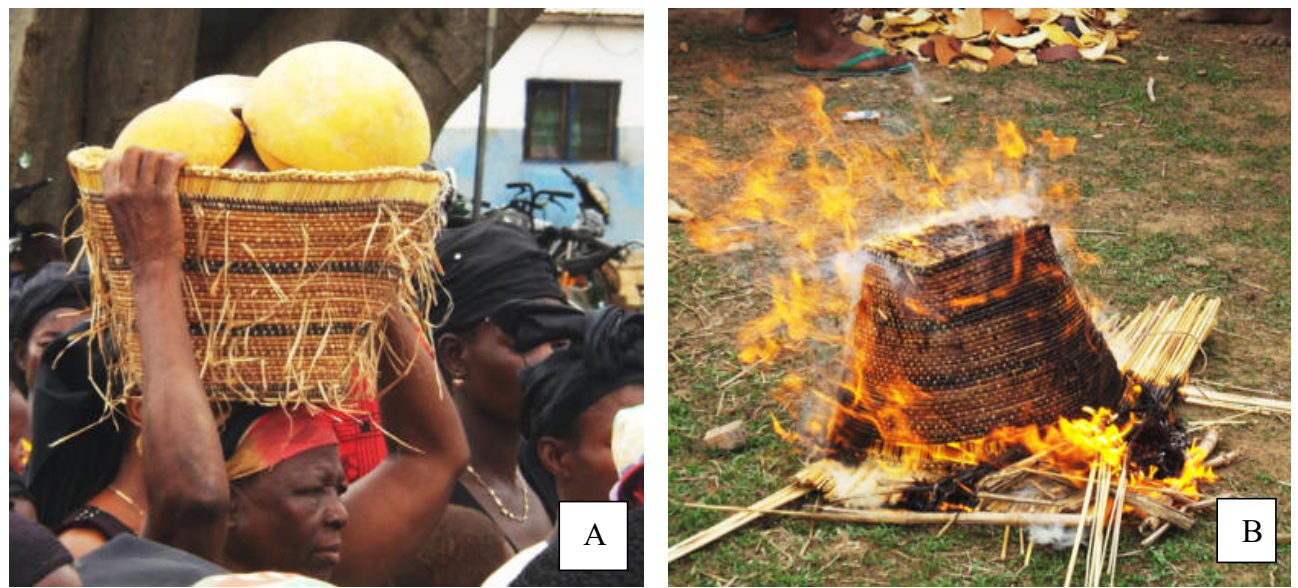

Figure 3. Kure pio being used to convey utensils for final funeral rites "A", kure pio being burnt during final funeral rites " $\mathrm{B}$ ".

Ga-ama Pio

The ga-ama pio utility container captured in figure 4 below is used for storing materials such as tobacco, groundnuts, money, and pieces of jewelry. The basket is similarly constructed as the pio baskets described above. The ga-ama pio, however, is integrated lavishly with leather materials and mounted on four vertical stands affixed to the base of the article. The container has a leather lid, which is stretched over the rim of the basket. The rim of this basket measures $28 \mathrm{~cm}$ in diameter. The basket has slanted sides, which tower to a height of $26.5 \mathrm{~cm}$, and a square base measuring $22 \times 22 \mathrm{~cm}$. There are braided leather cords affixed to the body of the basket, which aids in securing the lid onto the rim of the basket. The body of the basket is also decorated with braided leather accessories as evident in the article shown below.

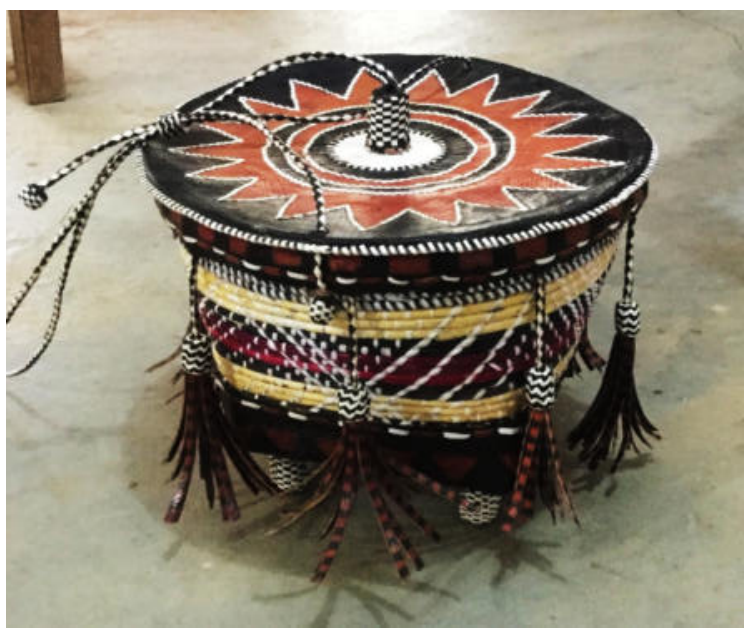

Figure 4. Ga-ama Pio

\subsubsection{Piog/Benka Baskets}

The piog basket, which is also called benka (See figure 5), is mostly woven by men. Three different sizes of the basket were seen during the study. The largest of the three, has a round base, measuring about $35 \mathrm{~cm}$. The slanted sides reached a height of $40 \mathrm{~cm}$, and the rim's diameter is $50 \mathrm{~cm}$. The medium size variety has a base diameter of $28 \mathrm{~cm}$, a height of $37 \mathrm{~cm}$ and a rim diameter of $34 \mathrm{~cm}$, while the smallest of the three baskets has a base of $23 \mathrm{~cm}$, a height of $26 \mathrm{~cm}$, and a rim diameter of $25 \mathrm{~cm}$. The Piog baskets, as articulated by the respondents, are employed in diverse spheres of the Gurene society. It is used to cart dried clay, loam and cow dung when constructing houses. They are equally employed as harvesting and storing baskets, beverage strainers, and also used by market women to sell assorted items in the market. 


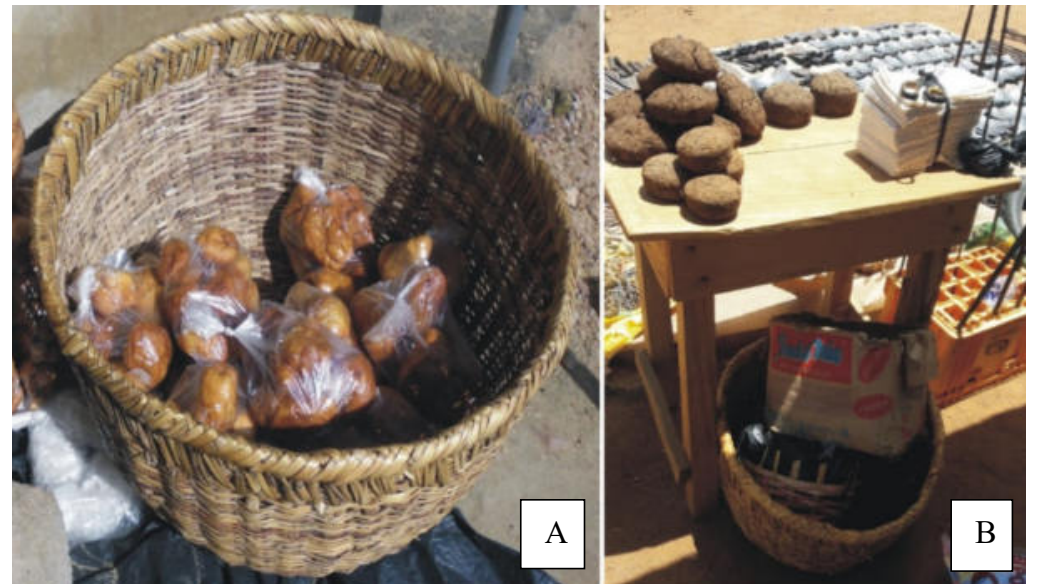

Figure 5. Piog/benka baskets being used to sell items in the market

\subsection{Architectural Basketry Objects}

Three assortments of architectural basketry articles were observed during the study. They comprised conical granary roofs (za'anu, sompeko or tampeko) and two mat varieties, viz; kpaska/mopele and zana mats accordingly. The articles are individually described below.

\subsubsection{Conical Granary Roofs (Za'anu)}

The article pictured below in figure 6 is variedly called za'anu, sompeko, or tampeko by the Gurene people. They are ubiquitous articles found in most indigenous Gurene communities. They function as roofing caps for earth-built granaries, which are mainly used for storing grains. The articles have diameters that ranged between $90 \mathrm{~cm}-120 \mathrm{~cm}$, and towers between $55 \mathrm{~cm}-69 \mathrm{~cm}$ in height. These products are usually crafted by men.

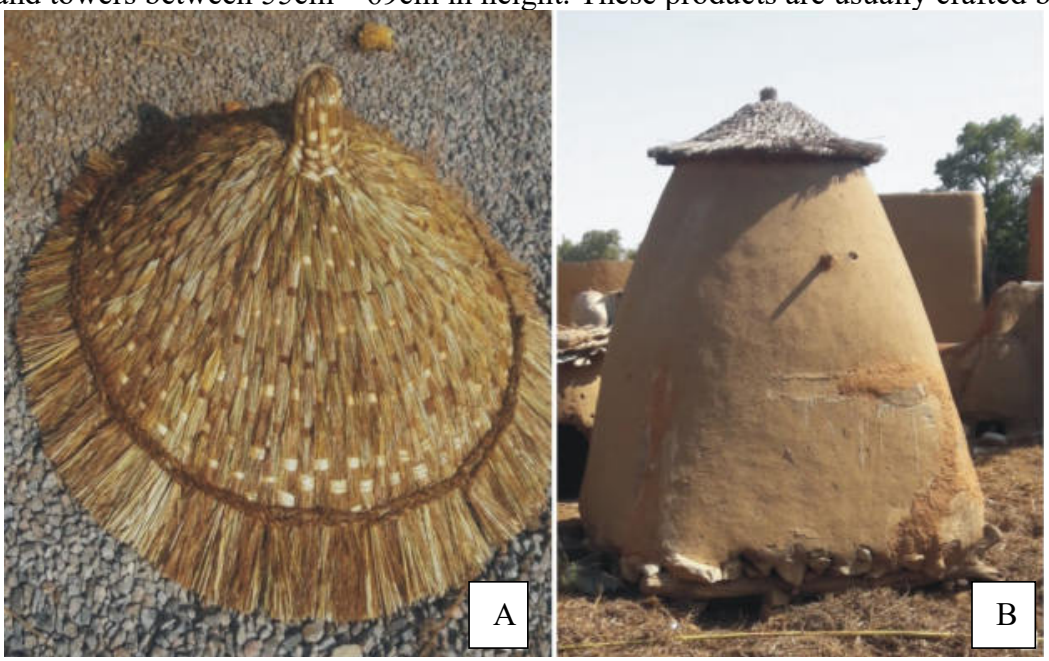

Figure 6. "A" Sompeko/ tampeko/ za'anu, "B" granary roof in use

\subsubsection{Roofing and Fencing Mats}

Two types of mats namely; gbaala (also known as kpaska or mopele) and zana were detected during the research.

Gbaala (Kpaska or Mopele)

Generally, gbaala mats (see figure 7 "A") are mostly woven by men. The mats are mainly used in roofing indigenous architectural structures such as sheds and earth-built houses. They are also employed in constructing fences. The mats studied have widths ranging from $130 \mathrm{~cm}$ to $280 \mathrm{~cm}$, and heights ranging from $150 \mathrm{~cm}$ to $200 \mathrm{~cm}$.

\section{Kpaha (Zana)}

Kpaha, which is also called zana (see figure 7 "B"), is produced by both sexes. These light-weight mats are used in various applications. They are utilized as sun-shields, as sleeping mats, as fencing mats, and as roofing materials. The sizes of the mats studied range from $85 \mathrm{~cm}-160 \mathrm{~cm}$ in widths, with lengths ranging between 

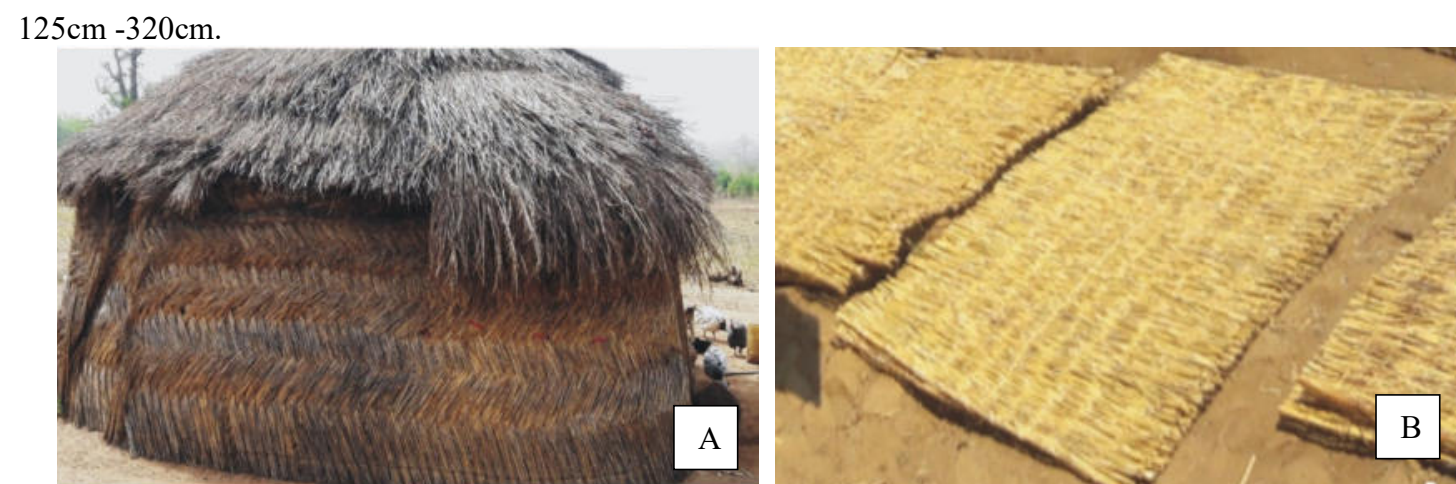

\subsection{Hats}

Figure 7. Gbaala/ Kpaska/ mopele mats being used as fence "A", kpahal zana mat "B"

Hats are generally called sepibre or sepi among the Gurene people. Five assortments of hats were seen and studied during the research period. Hats are widely worn in the Upper East Region, especially during the dry and hot season, which spans mid-October to May.

The hat shown in figure 8 "A" is known as rasta hat. The hat has twisted grass fibres attached all over the outer brim and crown of the hat. The crown is conical in shape, and has a loop crafted at the apex of the crown. This hat, according to the respondents, is mostly worn by young men when attending festivals and funerals. The diameter of this type of hats range between $29-38 \mathrm{~cm}$, and the height varies between $23-31 \mathrm{~cm}$.

Figure 8 "B" pictures the most popular variety of hats, which is patronized by the indigenes and outsiders alike. The hat, which is locally called Sepi ka'ate, may be worn by both the young and old, to funerals, festivals, the farm, or as a general dressing hat. The diameter of Sepi ka'ate normally ranges between $30-39 \mathrm{~cm}$, and the height of the crown varies between $14.5-18 \mathrm{~cm}$.

3.3.1 Sepi billa is the smallest of all the hats and is shown in figure 8 "C". It is used as an everyday hat, mostly to farm. Some informants explained in recent times, it is being used by the youth as a dressing hat. The diameter of these hats ranges between $28-31 \mathrm{~cm}$, and the height of the crowns varies between $14.5-18 \mathrm{~cm}$.

3.3.2 Akata-pore, is the name of the hat shown in figure 8 "D". These varieties of hats are usually crafted with a hole at the centre of the crown. The hats have series of twisted grass fibres arranged around the brim. They may be used by both the young and old during hot sunny days to festivals, funerals, and as everyday dressing accessory. The diameter of these type of hats ranges from $30-35 \mathrm{~cm}$, and the height of the crowns range from $14.5-19 \mathrm{~cm}$.

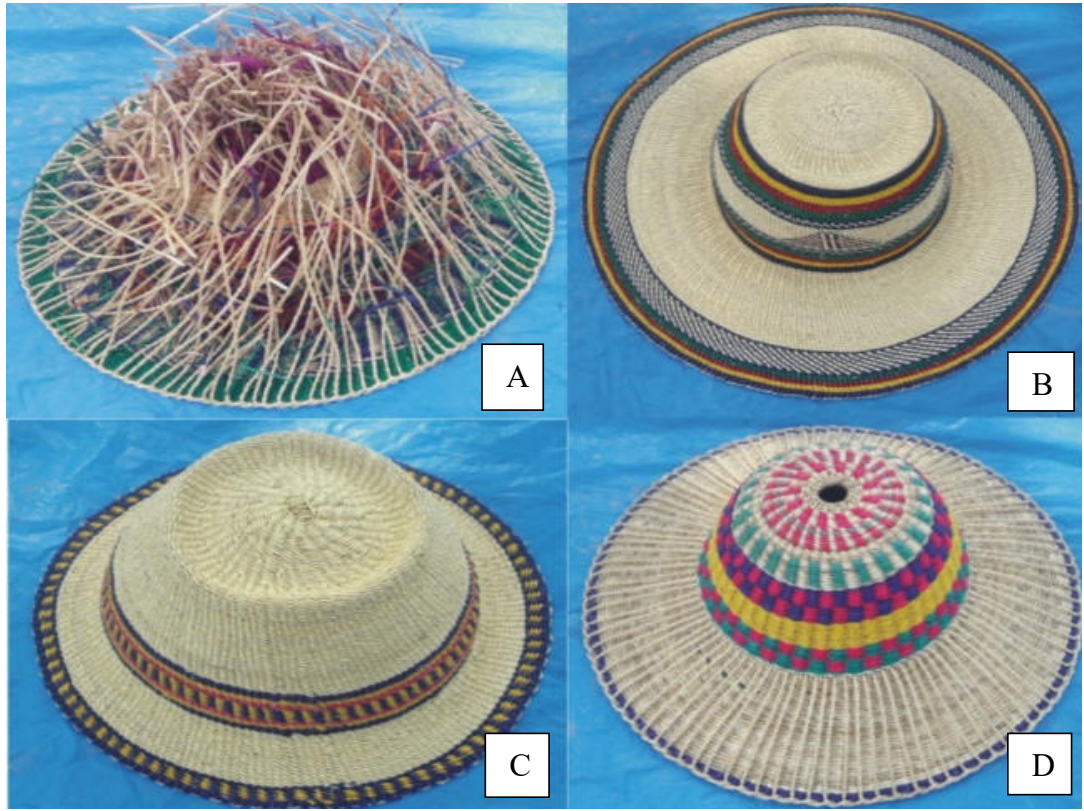

Figure 8. Hats: Rasta hat "A", Sepi ka'ate "B", Sepi billa "C" and Akata-pore "D" 


\subsubsection{Sepibre}

The hat depicted in figure 9 below is called sepibre. It is mostly used by market women and food vendors who ply their trade under the scorching sun. The hat has wide brims and deep crowns, which could balance comfortably on the head of women. The diameter of these hats mostly varies between $62-77 \mathrm{~cm}$ and the height of the crowns from the brim usually range between $18-25 \mathrm{~cm}$.

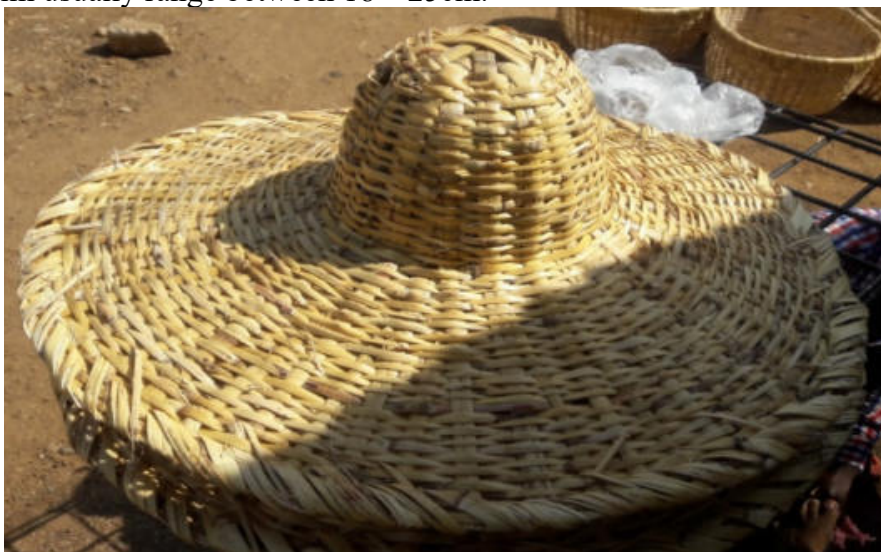

Figure 9. Sepibre

\subsubsection{Noyo and Sawula Hats}

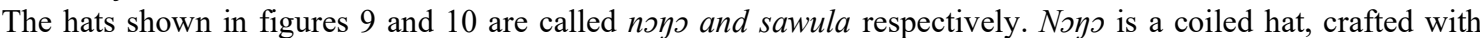
sheepskin affixed to the crown of the hat. The rim of the article is bound with black leather. The hat has braided leather cords attached to the rim of the hat. It measures $22 \mathrm{~cm}$ in diameter and stands at $19 \mathrm{~cm}$ (excluding the sheepskin accessory).


Figure 10. Nэyว "A", a man wearing $n э \eta$ "B"

The sawula hat (see figure 10) is equally a coiled object. The crown of the hat is appended with two cow horns, while the outer sides of the hat are decorated with red velvet, cowries, and black leather. The sawula hat has braided leather cords attached to the velvet decorations of the hat. The rim of this hat is also bound with black leather.

These hats are important cultural items used by men during the performance of final funeral rites of deceased relatives. They are used in special ceremonial dances. The respondents explained that the dances are symbolic performances, which seek to unveil the root cause(s) of the death of a deceased person and fight it. It was further explained that the Gurene people view death as an oppressor and for that matter, an enemy, that needs to be fought and vanquished.

A section of the respondents also articulated that nэฤว and sawula were in times past imbued with portent spiritual powers that protected warriors during battles. The modern-day performances, according to the respondents, were therefore enacted in memoriam of their courageous ancestors whose brave efforts liberated the 
Gurene people from their persecutors.

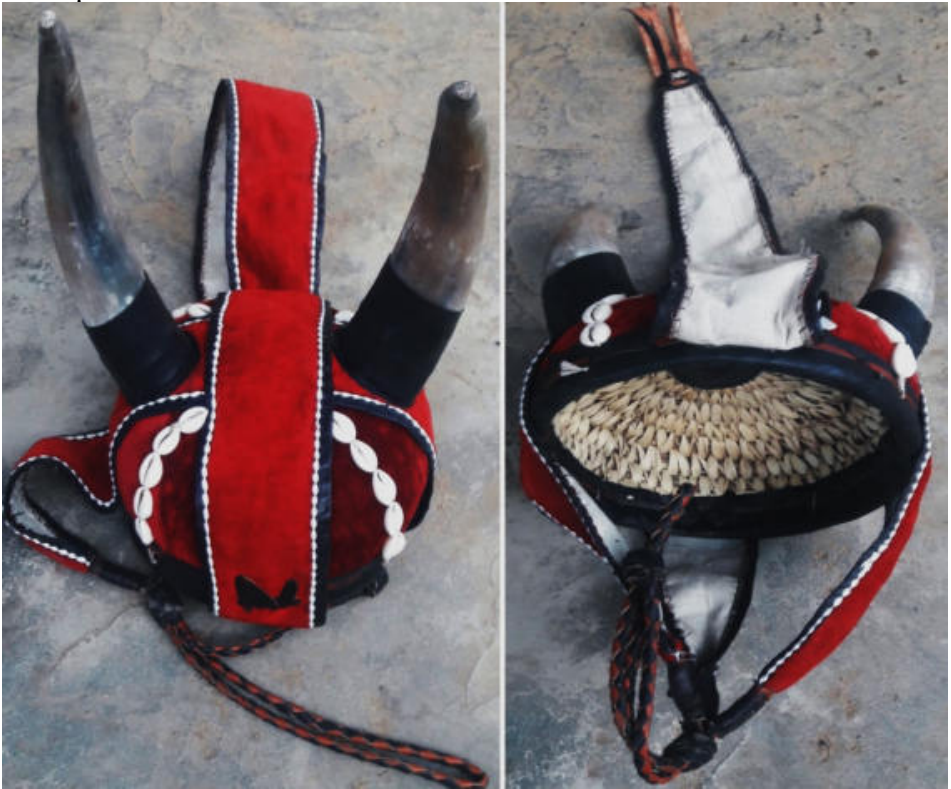

Figure 11. Sawula

\subsection{Feminine Clothing Articles}

Two different clothing articles were discovered and studied during the research. These objects, which are called valeya/sapole and voys/voneya, are presented below in figures 12 and 13 respectively.

\subsubsection{Valeya/ Sapole}

The article captured below in figure 12, according to a section of the interviewees, is one of the oldest basketry objects in the Gurene community. The object, which is called valeya (plural - valesi) or sapole, was widely worn by women around their waists in the olden days, to anchor another basketry object called voys (described in figure 13 below) and a textile piece (which falls outside the scope of the current study), to shield their anal and genital regions. It was also explained that the valeya could be spiritually energised by medicine-men or herbalists for use as protection against malevolent spirits, witchcraft, and barrenness. The respondents avered that in contemporary times, valeya is mainly used together with two other accessories (mentioned earlier) to dress elderly deceased women for burial. The informants indicated that some elderly women still use them in their day-to-day activities.

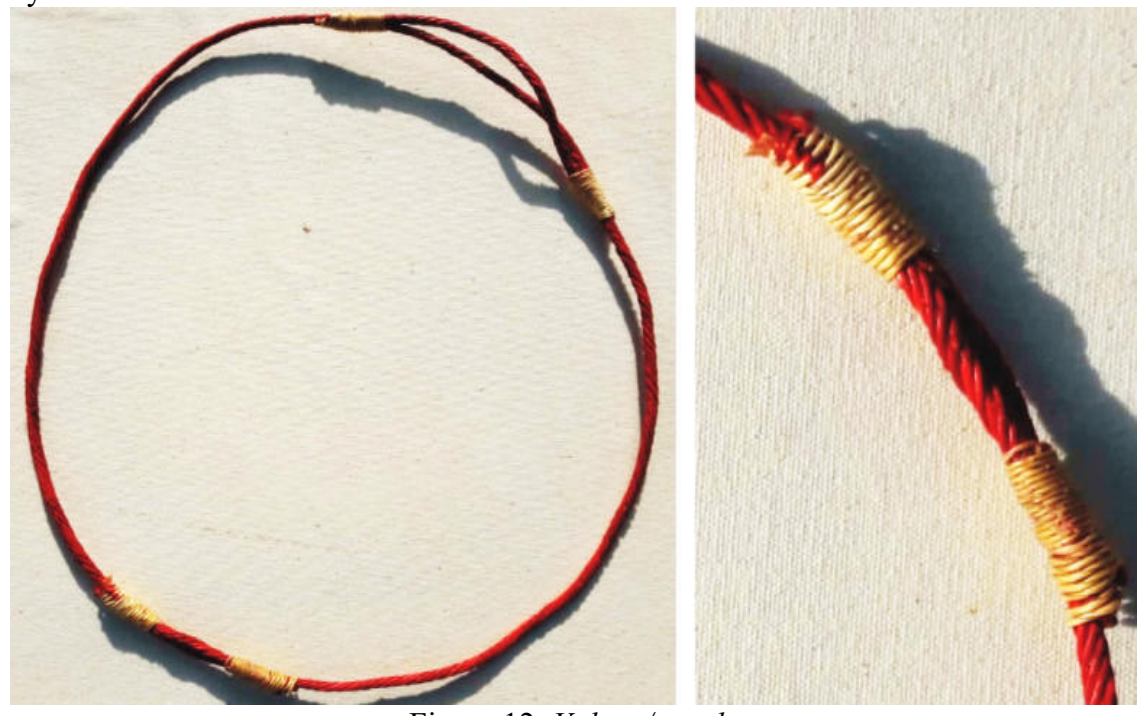

Figure 12. Valeya/sapole 


\subsubsection{Vэทว /Vэnеทа}

The artefact pictured in figure 13 below is known among the Gurene people as vəy or voneya. The object is made of braided and twinned kenaf fibers, and were used together with valeya (shown in figure 12 above) by women in times past as daily clothing articles. They were mostly used to cover the anal regions. Currently, vons is used together with valeya and another accessory (which is outside the scope of this study), to dress elderly deceased women for burial.

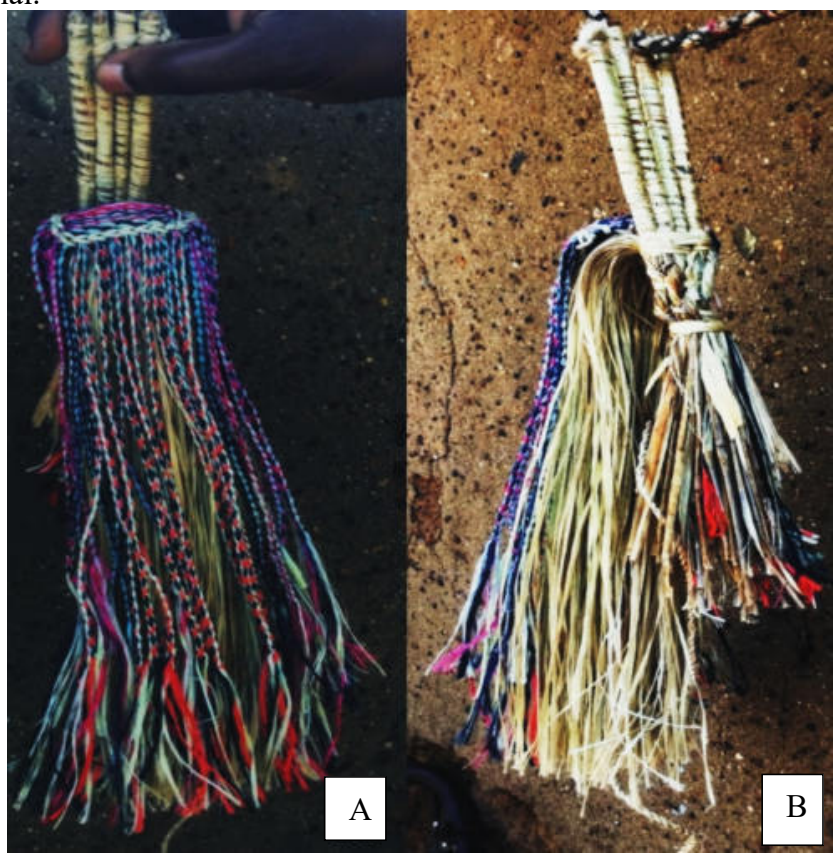

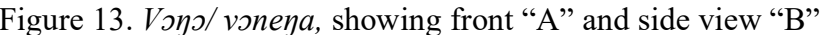

\subsection{Rattles (Sinyasa/ Seyaka)}

Dancing and singing are integral aspects of the Gurene people. Rattles, which are indigenously called senyasa or seyaka, are widely used mostly by women as musical accompaniments in the performance of songs. The rattles, which come in various colour combinations, are used during festivals, naming and marriage ceremonies, funeral rites, and various church programmes.
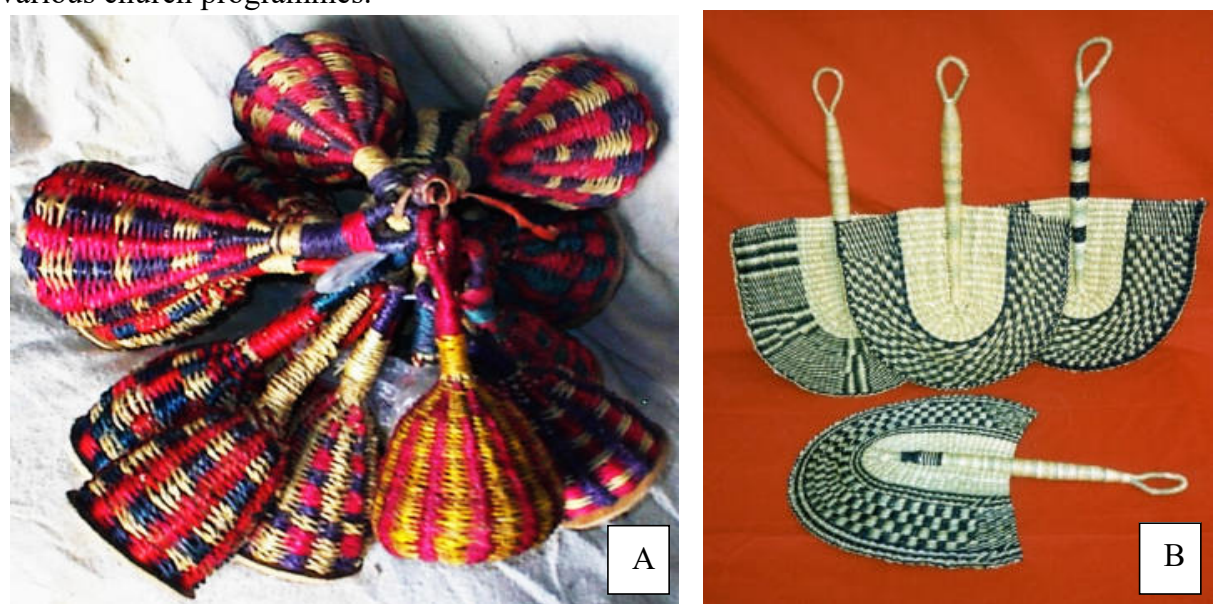

Figure 14. Rattles (Sinyasa/seyaka) “A” Fans (peyJ/peno) “B”

3.6 Fans (Pengo)

Gurene fans (see figure 14 "A") are intricately woven articles which come in different colour combinations. The fans are important cultural objects which are used widely in the Gurene society. Generally, the average temperature in the Upper East Region of Ghana can exceed 41 degrees Celsius, especially during the dry season, which spans mid-October to May. The fans are therefore used extensively around this period by many to cool themselves. The respondents explained that the fans are also used to cool tribal chiefs and Tindaanas (land 
custodians) by their attendants whenever they are seated. Some of the respondents also averred that the fans are essential in driving away mosquitoes at night, and flies during the day time. It was also explained that when preparing corpses for burial, the daughters of the deceased person utilizes this article to fan the corpse. This action, the respondents explained symbolizes love, respect, and affection for the deceased.

\subsection{Sleeping and Burial Mats}

Sleeping and Burial Mats

The mats shown in figure 15 are variedly called songo, səy, spelegu or sumpele by the Gurene people. It was explained that the mats may be used for sleeping or burial activities. A section of the respondents explained that mats meant for burial activities were usually produced with red and black dyed reed materials mixed with the naturally-coloured ones, as pictured in figure 15 "A" below. Other respondents also averred that the plainlywoven mats (mats produced without dyed reed materials) could sometimes also be used for burial ceremonies. The burial mats, as pointed out by some respondents, could be used in both indigenous and Christian burial activities.
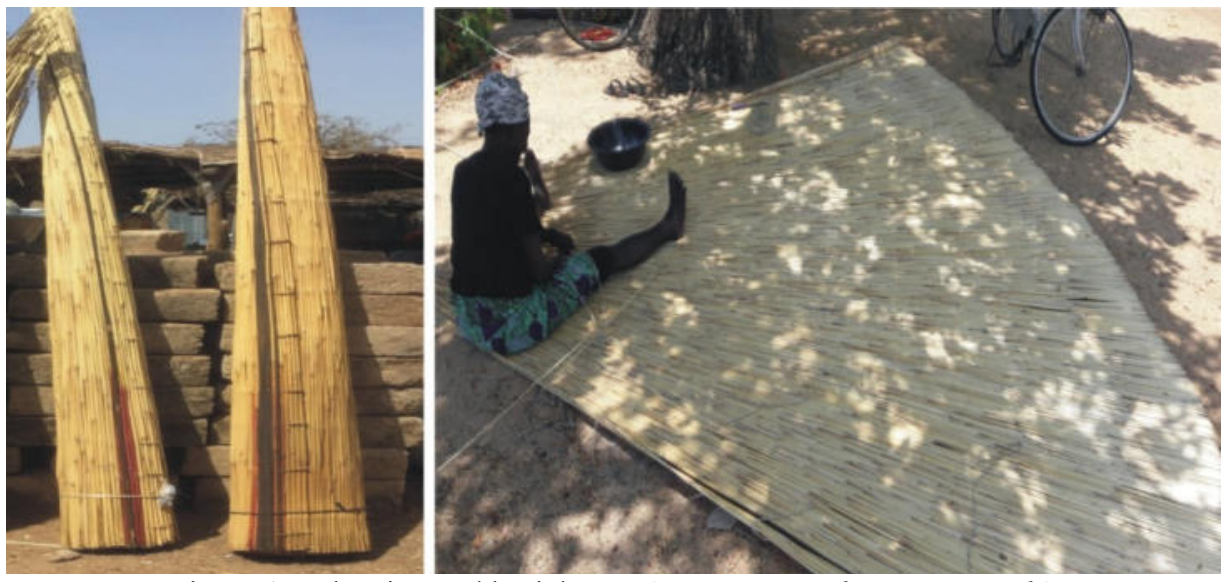

Figure 15. Sleeping and burial mats (sonyっ, səy, pelegu or sumpele)

\subsection{Cages and Nests}

Four assortments of cages and nests were observed during the study. These basketry articles are pictured in figures 16, 17 and 18 below.

The basketry article pictured in figure 16 " $\mathrm{A}$ " below, is normally used in many Gurene communities as coop for young chicks and guinea fowls at night. This circular basketry ware has a conical top, and an aperture crafted on the body of the basket to enable the entry and exit of the chickens and guinea fowls. The article could be woven to various specifications. The sizes of those studied have diameter ranges of between $45 \mathrm{~cm}-68 \mathrm{~cm}$, and towers to heights of between $34 \mathrm{~cm}-52 \mathrm{~cm}$.

The cage shown in figure 16 "B" is a circular object with a lid. The ones seen during the study have widths of between $34-66 \mathrm{~cm}$ in diameter, and height variations of between $32-46 \mathrm{~cm}$. This product is normally used to transport chickens and guinea fowls to the market to be sold. The respondents added that when members of a household are going to the farm, they sometimes use this cage to convey young chickens and guinea fowls to the farm for them to be fed with various insects and worms that would be unearthed during tilling. The cages, the respondents explained, are also used to protect the birds from hawk attacks. 


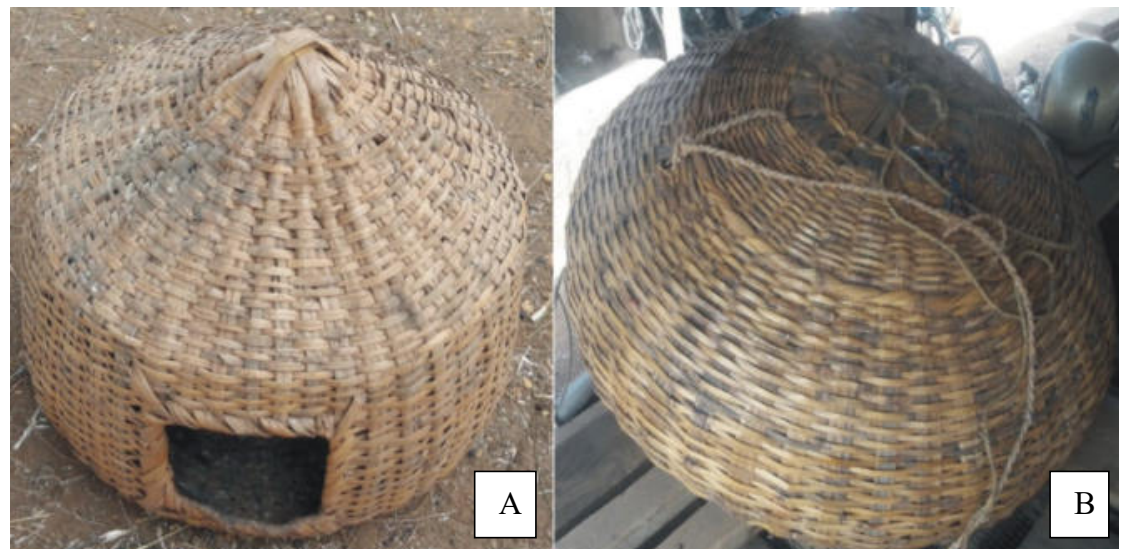

Figure 16. Koysnko cages

The basketry item shown in figure 17 " $\mathrm{A}$ ", is an oblong-shaped object, which is mainly used as a nest for birds such as doves. They are mostly hanged below the roof of a building, to enable birds access it. Some respondents explained that this basketry object could also be used to transport birds to the farm. They may also be used to cart various food items to the farm by affixing a shoulder sling and a lid to it, as shown in figure 18 "B" below.

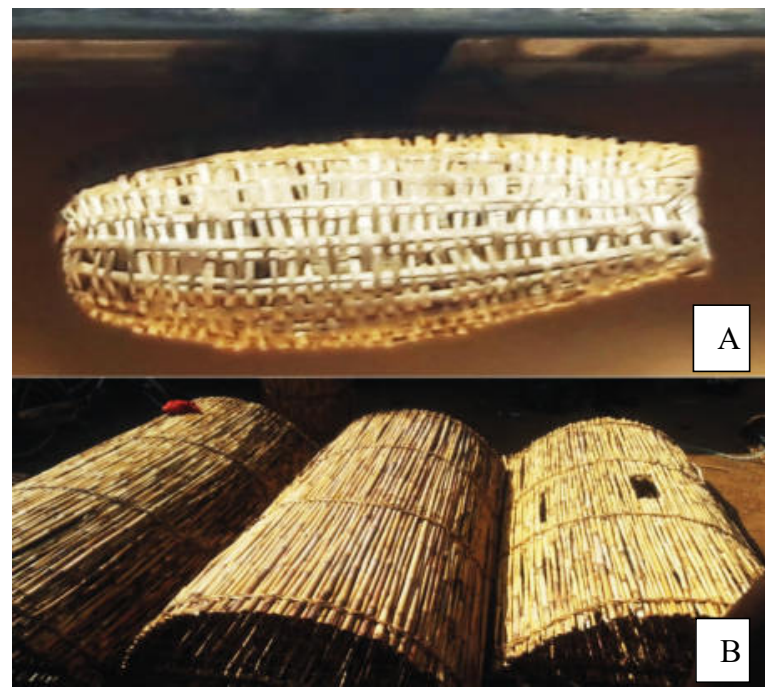

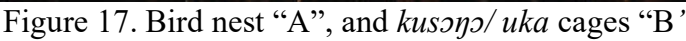

Figure 17 "B" captures the largest of all the cages studied. This cage is locally called kuson or uka. The main body of the cage is constructed around a D-shaped wooden frame, with netting barricades at both ends. Cross sectionally, the cages have semi-circular appearance. This variety of cages, as expained by the respondents, are used mainly to transport guinea fowls, chicken and ducks to market centers for sale on bicycles or motorbikes, as shown in figure 18 "A" below. Various sizes of this cage were seen during the study. The lengths range between $55-130 \mathrm{~cm}$, the widths vary between $50-85 \mathrm{~cm}$, and their heights vary between $32-$ $56 \mathrm{~cm}$. 


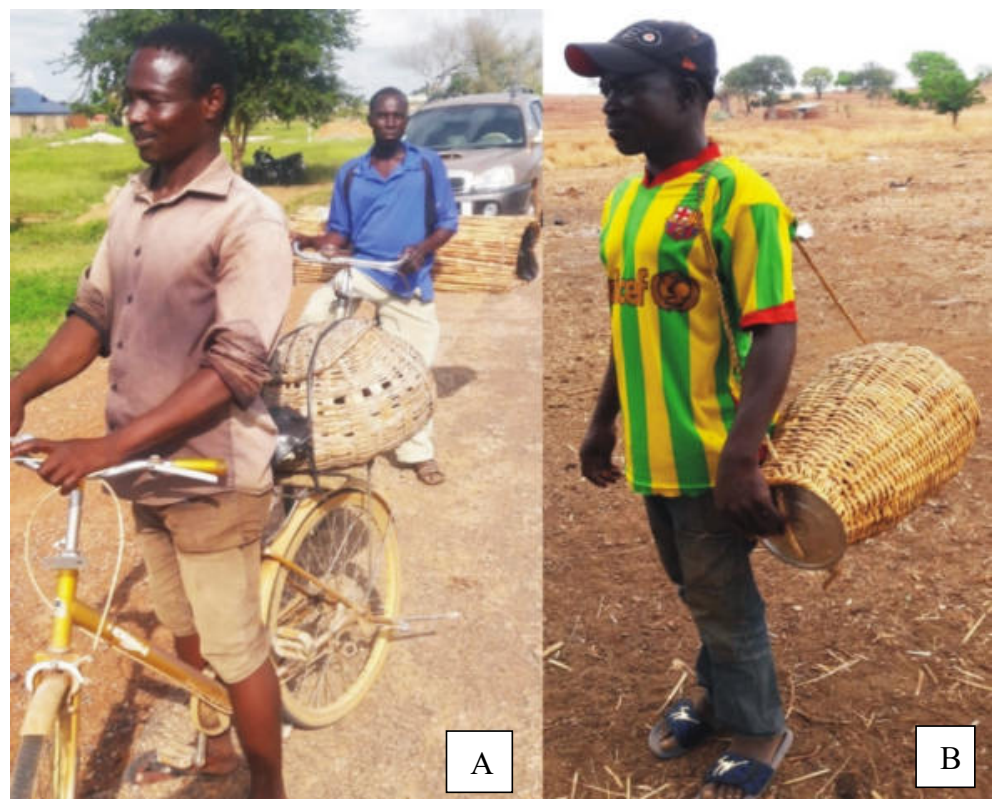

Figure 18. A Cages being carried on bicycles "A", and a nest being used as a carrier "B"

\section{Discussions}

The study revealed a variety of Gurene basketry articles which are manufactured distinctly to promote comfortable day-to-day activities in the Gurene society. It was noticed that some of the basketry objects serve multiple purposes, just as those produced and used in other African countries, as documented by Silva (2011). Some of the multifunctional basketry objects observed and studied are; pio baskets (figures 2 "A" and "B"), piog baskets (figure 5), gbaala, kpaha, and sony mats (see figures 17 "A" and "B" as well as figure 15), voyo/voleya clothing articles (figure 13), valena/sapole clothing articles (figure 12) as well as bird nests (figure 17 " $\mathrm{A}$ ").

The functions of the various mats discovered in the Gurene society mimics those found in other ethnic societies in Ghana and some other African societies. For example, Japan Association for International Collaboration of Agriculture and Forestry's (2010) study, which sampled information from Accra, Takoradi, Tarkwa, Faowanye and Keta, all in Ghana, found out that mats, apart from being used as bedding materials, were employed as sun shields, just as zana mats are used in the Gurene society. Also, Biro (2011) found out that Tutsi people of Rwanda and Burundi, just as their Gurene counterparts, extensively used various mats as architectural supplements. The use of sonys mats in funeral activities in the Gurene culture equally resonates with similar practices among the Weh of Cameroon, who according to Geary (1987), used mats in preparing corpses for burial activities.

The results of the study bore witness to the fact that basketry objects such as; the various pio and piog/benka baskets, as well as vəэว and valeya clothing objects, are the key basketry articles associated with Gurene womanhood. While the pio and benka baskets aid in the cultivation, harvesting, carting, storing, and the preparation of food items towards the sustenance of families in the Gurene society, voys and valeya could be seen as articles that define and preserve feminine dignity in the Gurene culture. It is therefore not strange that kure pio baskets, as well as vəyว, and valeya clothing items play definitive roles in the funeral rites of elderly Gurene women.

It is also important to note that the use of vəyว as clothing article among Gurene women, resembles similar usage of a basketry object among the Weh people of Aghem-Fungom neighbourhood of Cameroon, known as kesunngi (Geary, 1987). Geary writes that the kesunngi was worn around the waist, towards the back to conceal the buttocks, just as vəэo is used by Gurene women.

Generally, some of the articles observed during the study fits the description of basketry objects discovered by Smith (1978) during his study among the "Gurensi", "Tallensi" and "Nabdam" communities of the Upper East Region of Ghana. Smith wrote about "flat-bottomed cane baskets", "bulbous baskets", "semi-circular fans", "wide-brimmed hats" and "rattles" which were produced and used by the Gurene people. This clearly shows that some of the traditional basketry forms known among the Gurene populace in the 70 s are still being crafted and used in modern times.

The utilization of sony mats as burial articles could be a very practical and inexpensive way of interring the dead, especially in a society where poverty is endemic. It is the contention of the researchers that the use of kure pio baskets in the funeral rites of elderly deceased women revealed the Gurene people's belief in life after 
death. This is so because, the rites involving the kure pio basket, as intimated by the informants, is intended to transfer the kure pio basket and other culinary objects such as pots and calabashes to the deceased woman for use in the spirit world. This clearly suggests that the Gurene people believe life still exists after death. It is equally important to mention that the symbolic dances performed during final funeral rites of deceased relatives by Gurene men with the aid of noys and sawula hats in the Gurene society, again brings to the fore the Gurene people's notion about death and its attributes.

\section{Conclusions}

The study unearthed a wide variety of Gurene basketry articles which are employed in various sectors of the Gurene society. The study shows that basketry articles are still relevant in the daily activities of the Gurene people, despite the abundant presence of various plastic, wooden, paper, and metallic containers, and carriers in the Gurene society. Generally, the findings of the research demonstrate that basketry articles are utilized in harvesting, carting, and storing of various farm produces in the Gurene society. The study equally shows that some of the articles are used in funeral rites. They are also employed as; sleeping mats, cages, nests, fans, hats, rattles, and clothing items. A given number of the basketry articles are applied in winnowing activities, and equally used as architectural accessories, beverage strainers, and as containers which are used in selling assorted products in the various Gurene markets.

The research reveals the enormous socio-cultural contributions of basketry in the Gurene society. This delineates the basketry sector as a very important area worth developing, to preserve the artistic heritage and maximize the positive effects of the sector. There is the need for the establishment of a basketry arts demonstration village in the Bolgatanaga municipality. The basketry village, which should be populated with expert craftsmen and women, could offer practical lessons in the production of the various basketry articles found in the Gurene community. The basketry crafting lessons could be extended to tourists, students, researchers, and basket lovers at a fee. This would help in generating extra income for the artisans, preserve the basketry crafting tradition for posterity, boost tourist inflow to the area, and enable people to learn about the basketry arts of the Gurene people.

\section{References}

Asante, B. (2005). Women's craft guilds and the traditional basketry (ge mot) of Harare, Ethiopia. African study monographs $29,61-72$

Balfet H. J. (2014). Basketry in: Encyclopedia Britannica. Encyclopedia Britannica Inc.

Biro, Y. (2011). Tutsi basketry. Retrieved from: http://www.metmuseum.org/toah/hd/tuts/hd tuts.htm (Accessed December 9, 2016).

Bizzari, H. B. (2012). Weaving and basketry in ancient and modern Egypt. Retrieved from http://www.touregypt.net/featurestories/basketry.htm on 30 November 2016.

Bunn S. (2014). Woven Communities: Exploring the Dynamics of Socio-cultural Change through Material Culture, Special Case Study on Scottish Vernacular basketry. Connected Communities, St Andrews.

Crowford, B. J. (2003). Basketry: 18 easy and beautiful baskets to make. Lark Books, New York.

Geary C. (1987). Basketry in the Aghem-Fungom Area of the Cameroon Grassfields. African Arts, 20, 3, 42-53 - 89-90.

Harvey, V. I. (1975). The techniques of basketry. B.T. Batsford Ltd, London.

Japan Association for International Collaboration of Agriculture and Forestry (2010). Fiber Plants of Africa and their Usage. Retrieved from http://www.jaicaf.or.jp/fileadmin/user_upload/publications/Fiber_e_1.pdf. on 3 April 2018.

Kaller B. (2012). The art of producing sustainable consumer goods: basketry. Retrieved from: http://www.lowtechmagazine.com/2012/02/basketry-the-art-of-producing-sustainable-consumer-goods.html on 6 August 2016.

Kepe T. (2003). Use, control and value of craft material - Cyperus textilis: Perspectives from a Mpondo village, South Africa. South African Geographical Journal 85,152-157.

Levinsohn R. (1980). Basketry: A Renaissance in Southern Africa. African Arts, 13, 4, 17-19.

Ljunggren, I. (2007). Weaving a way out of poverty, basket weaving as poverty reduction strategy among rural female poor in Bolgatanga, Northern Ghana.

Retrieved from http://www.rucsdigitaleprojektbibliotek.dk/bitstream/1800/2507/3/z0.pdf on 3 April 2018.

Ng, F.M.C. (2004). A review of the development of basketry, braiding, crochet, and felting towards the creation of seamless fashion. Research Journal of Textile and Apparel 8(2), 65 - 75.

Pollock P. (1993). Basketry: Tradition and Change. Journal of Museum Ethnography 4, 1-24.

Silva, S. (2004). Basketry: Africa. In Philip M. Peak and Kwesi Yanah (eds.) African Folklore: An Encyclopaedia. Taylor and Francis, Abingdon.

Singh, D. J. \& Davidson, J. (2016). Learning basket weaving - traditional and modern techniques and methods. JB-BIS Publishing, Mendon.

Wendrich, W. (1991). Who is afraid of basketry: a guide to recording basketry and cordage for archaeologists and ethnographers. Leiden: Centre for Non-Western Studies, Leiden University. 